\title{
Free Space Optical Communication: The Last Mile Solution to High Speed Communication Networks
}

\section{Miglani R*}

Department of Communication System, Lovely Professional University, Punjab, India

\section{Introduction}

Free space optical communication is a line of sight based communication which requires information carrying light to travel across the atmosphere between the transmitting and receiving nodes. The earliest form of light based communication through free space can be traced back to 1880 when Alexander Graham Bell, demonstrated the working of a photo phone, which could carry voice signals modulated on sun's radiation through a distance of 200 meters. However at that time, the equipment and methodology involved was very nascent, thus this experiment was not any major success. However, in late 1970s with advent of advanced optical devices like high powered tuneable lasers and detectors, FSO communication has witnessed massive upgradation along with huge interest of researchers around the globe. The first commercial FSO link was used in Japan by Nippon Electronic Company in 1970s to handle air traffic.

Till date FSO technology has been used to fulfil the requirements of military for faster and discrete communications. Space research agencies like NASA has also been vigorously working on light based deep space communication which will be used for various space expeditions like that of mars and lunar probe missions.

In spite of these initiatives, FSO could not really pick up commercially because our need for reliable communication networks was adequately satisfied by the existing radio and microwave infrastructure. Secondly, FSO being light based communication through free space was always an unreliable communication technique, especially under the conditions of fog, rain, snow and atmospheric turbulence effects which leads to scattering and loss of optical signal. These two were major reasons that lead to slow penetration of FSO links into the commercial domain.

However in current scenario where we are facing severe bandwidth crunch and the demand for data rates at all-time high, FSO systems can be an excellent alternative to established RF system for data and voice communication. Since FSOlinks operate in licence free band, hence these systems can be very conveniently used for short range communication, particularly as last mile solution to bridge the bandwidth gap between the end user and the network hub. Despite atmospheric effects being biggest challenge in the way of FSO implementation, the hybrid version of FSO and RF has seen great experimental success and will surely be the future practical solution to migrate from current bandwidth starved RF systems to optical systems along with maintaining $99.999 \%$ uptime. As explained in the introductory part, FSO systems as line of sight based optical communication systems the block diagram as shown in Figure 1 will bring out more clarity.

\section{Overview of FSO links in contrast to RF networks}

The principal advantage of FSO technology is very high bandwidth availability, which could provide broadband wireless extensions to Internet backbones providing service to end-users. This could enable the prospect of delay-free web. browsing and data library access, electronic commerce, streaming audio and video, video-on-demand, video teleconferencing, real-time medical imaging transfer, enterprise networking and work-sharing capabilities, which could require as much as a $100 \mathrm{Mbps}$ data rate on a sustained basis (Table 1).

Certain set of advantages make FSO communication systems as an undoubted winner over RF based communication model, these have been discussed below:

(a) Spectrum licensing: The biggest concern today for radio systems is interference with existing commercial and military systems. Currently, the frequencies above $300 \mathrm{GHz}$ (less than $1 \mathrm{~mm}$ in wavelength) have not been regulated by FCC. Free Space Optics (FSO) systems use frequencies above $3 \mathrm{THz}$ so they do not require spectrum licensing.

(b) Bandwidth and data rates: Currently, commercially available FSO products provide data rates of $2.5 \mathrm{Gbps}$. Demonstration systems report data rates as high as $160 \mathrm{Gbps}$. Bandwidth is closely related to data rate. High data rates require a lot of spectrum, which is why FSO technology is becoming more popular.

(c) Data security: In order to detect a laser transmission, a detector/infrared viewer or receiver must be located within the laser beam cone, between the transmitters or behind the receiver to intercept uncollected part of the beam, but this is highly unlikely because microwave transmitters have a divergence angle of a few degrees where FSO systems have a few milli radians ( $1 \mathrm{mrad}=0.0573$ degrees $)$. The interruption of the laser signal would result a sudden drop in received power at which point the manager software built in the systems may notice and cease the transmission, which may not be sufficient to extract information.

\section{Limitations of FSO Links}

In spite of some major advantages, FSO links have certain inherit limitations which have been listed below

\begin{tabular}{|c|c|c|}
\hline & FSO LINKS & RF LINKS \\
\hline Typical data rate & $100 \mathrm{Mbps}$ to Gbps & Less than $100 \mathrm{Mbps}$ \\
\hline Channel security & High & Low \\
\hline Component dimension & Small & Large \\
\hline Networking architecture & Scalable & Non-scalable \\
\hline Source of signal degradation & Atmospheric turbulence & Multipath fading and rain \\
\hline
\end{tabular}

Table 1: FSO links vs. traditional RF links.

*Corresponding author: Miglani R, Department of Communication System, Lovely Professional University, Punjab, India, Tel: 1800102 4431; E-mail: rajan.16957@lpu.co.in

Received July 31, 2017; Accepted August 06, 2017; Published September 01, 2017

Citation: Miglani R (2017) Free Space Optical Communication: The Last Mile Solution to High Speed Communication Networks. J Laser Opt Photonics 4: e112. doi: 10.4172/2469-410X.1000e112

Copyright: (c) 2017 Miglani R. This is an open-access article distributed under the terms of the Creative Commons Attribution License, which permits unrestricted use, distribution, and reproduction in any medium, provided the original author and source are credited. 


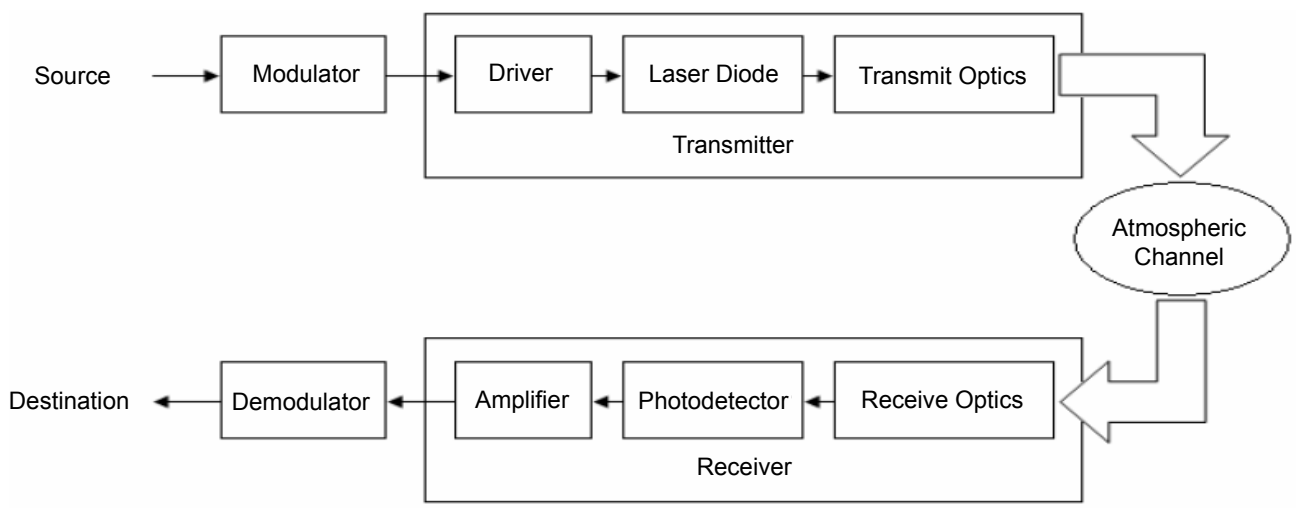

Figure 1: Subsystem showing functional parts of FSO system.

a. Atmospheric effects: Since FSO systems involves transmission of optically modulated signal through atmosphere as medium, hence the optical radiation is subjected to various atmospheric phenomena's like rain, wind, snow and fog etc. These effects along with atmospheric turbulence can make the communication link very inconsistent. Since atmosphere is composed of various gases, aerosol particles and water droplets, the optical signal undergoes various degrees of scattering and degradation as it transverses along the link range.

b. Line of sight: Second challenge in FSO links is line of sigh communication. FSO links require highly directional narrow laser beams to communicate and there must not be any physical obstruction between the communicating nodes else the data may be either partially or completely lost, which is defiantly highly undesirable.

c. Directional precision: Free Space Optics (FSO) systems use narrow laser beams. Typically, commercially available FSO systems have a beam divergence from 0.1 degrees to 0.3 degrees, which is very small compared to microwave systems. For a beam divergence of 0.1 degrees, the diameter of the laser beam is 1.74 meters at a distance of $1 \mathrm{~km}$.

d. Laser safety: The safety of FSO technology is often a concern, since it uses lasers for transmission. LASER is an acronym that stands for Light Amplification by Stimulated Emission of Radiation. The laser produces an intense, highly directional beam of light. The unprotected human eye is extremely sensitive to laser radiation and can be permanently damaged from direct or reflected beams.

\section{Motivation}

More recently penetration of mobile and data services into our daily lives has led to exponential rise in demand for higher bandwidth and seamless communication. The RF communication networks are finding it difficult to cope up with current surge in demand for enhanced data rates. This is primarily because of huge cost that is associated with setting of high capacity RF networks, right from the purchase of expensive spectrum to installing and maintaining the hardware.

Optical wireless communication has been known to mankind for centuries, be it war tactics of military posts, Alexander's photo phone or even more recently NASA's inter space satellite communication links. Because of its operating bandwidth, optical links by default have huge data and bandwidth capacity. The prime motivation was to chalk out plan such that seamless communication can be complimented with excess bandwidth, and this leads us to look forward to something which is better known as Free Space Optical communication.
Although FSO links outplays conventional RF links when compared in terms of technological benchmarks like data speed, security, privacy, cost etc, but still FSO links have long way to go before they can complement or even substitute the RF regime. The reason for this is the unpredictable behaviour of atmosphere which leads to channel fading and at times it may just paralyse the link.

\section{Why is FSO still a last mile solution?}

Also FSO systems can be particularly very helpful in locations like mountainous regions where fibre lying is very tough task, or even in busy metro cities where roads can't be dug. In such scenario FSO system can not only prove to be extremely helpful but also cost effective too. In event of occurrence of natural or manmade disasters FSO links can be used to provide immediate temporary connectivity without much of sophisticated hardware as it was done right after 9/11 attacks in America, where FSO links were installed to provide immediate connectivity to overlook rescue and search operations as RF network had collapsed. However, the FSO technology itself is still in incubation stage with lot of hurdles to overcome before it reaches to end users in every household. Atmospheric disturbances are something we as network designers cannot deal directly, however with advent of technology, high powered lasers have been developed which are capable of overcoming scattering and fading effects caused by atmospheric turbulences. 